\title{
MRI chemical shift imaging of the fat content of the pancreas and liver of patients with type 2 diabetes mellitus
}

\author{
JUN CHAI ${ }^{1}$, PENG LIU $^{1}$, ERHU JIN ${ }^{1}$, TIANHAO SU ${ }^{1}$, JIE ZHANG $^{1}$, \\ KAINING SHI ${ }^{2}, \mathrm{XU} \mathrm{HONG}^{3}, \mathrm{JIE} \mathrm{YIN}^{3}$ and HENGCHI YU \\ ${ }^{1}$ Department of Radiology, Beijing Friendship Hospital, Capital Medical University, Beijing 100050; \\ ${ }^{2}$ GE Healthcare China, General Electric Company, Beijing 100176; ${ }^{3}$ Department of Endocrinology, \\ Beijing Friendship Hospital, Capital Medical University, Beijing 100050, P.R. China
}

Received November 8, 2014; Accepted November 25, 2015

DOI: $10.3892 /$ etm.2015.2925

\begin{abstract}
The present study aimed to investigate the association between the content and distribution of fat in the pancreas and liver in patients with type 2 diabetes mellitus (T2DM). A total of 70 patients newly diagnosed with T2DM (T2DM group) and 30 healthy volunteers (normal control group) were enrolled in the present study. Dual-echo magnetic resonance (MR) chemical shift imaging was used to measure the fat content of the liver and the head, body and tail regions of the pancreas. In addition, the distribution of fat in the various regions of the pancreas, as well as the average fat content of the pancreas versus the liver, were compared. The fat content of the pancreatic head, body and tail regions of the T2DM group were $5.59 \pm 4.70,4.80 \pm 3.75$ and $4.89 \pm 3.86 \%$, respectively. The fat content of these regions in the normal control group were $3.89 \pm 2.47,3.30 \pm 2.11$ and $3.23 \pm 2.23 \%$, respectively. The average fat content of the pancreas was $5.19 \pm 3.75 \%$ for the T2DM group and $3.47 \pm 2.00 \%$ for the normal control group. The average fat content of the liver was $9.87 \pm 3.19 \%$ for the T2DM group and $7.24 \pm 2.38 \%$ for the normal control group. Therefore, the results from MR chemical shift imaging suggested that there were no significant differences in the distribution of fat between the pancreas of patients newly diagnosed with T2DM and that from the healthy population; however, the average fat content in the pancreas of the T2DM group was significantly higher $(\mathrm{F}=3.597 ; \mathrm{P}<0.05)$, as compared with the normal control group. In addition, there was no correlation between the fat contents in the pancreas and liver in patients newly diagnosed with T2DM and the healthy population.
\end{abstract}

Correspondence to: Dr Erhu Jin, Department of Radiology, Beijing Friendship Hospital, Capital Medical University, 95 Yongan Road, Beijing 100050, P.R. China

E-mail: erhujin@126.com

Key words: pancreatic fat deposition, type 2 diabetes mellitus, magnetic resonance chemical shift imaging

\section{Introduction}

Fatty acids produced by metabolism in the human body are predominantly stored in adipose tissue in the form of triglycerides (1). However, if the rate of fatty acid production overloads the bearing capacity of adipose tissue, it is heterotopically deposited in non-fat tissues and organs, including the liver, pancreas, myocardium and skeletal muscle (2). Heterotopic fat deposition is a common phenomenon encountered during the examination of medical images $(1,2)$. Fat deposition in the liver and skeletal muscle may induce insulin resistance and a rise in blood sugar levels, which in turn may lead to type 2 diabetes mellitus (T2DM) (3). Obesity, insulin resistance and various other metabolic syndromes are crucially involved in the occurrence and development processes of non-alcoholic fatty liver disease (4). In addition, $10-20 \%$ of patients with non-alcoholic fatty liver disease develop non-alcoholic steatohepatitis, which may lead to cirrhosis of the liver and liver failure (5). The pathological process of pancreatic fat deposition, which resembles hepatic fat deposition, may cause inflammation, resulting in fatty pancreatitis (6). Pancreatic fat deposition has previously been associated with fatty liver disease, insulin resistance and metabolic syndromes $(7,8)$. In addition to insulin resistance, an insufficiency in the function of the islet cells may induce lipotoxicity injury, which has also been associated with the occurrence of T2DM (9).

Magnetic resonance (MR) chemical shift imaging is a novel method for the quantification of adipose tissue, and has a higher accuracy compared with MR spectroscopy, as it is not affected by the uniformity of the magnetic field (10). In addition, in a previous study, MR chemical shift imaging was easy to operate and the results were stable (10). The fat content of the pancreas in the normal population has previously been shown to increase with age; however, no significant difference in the distribution of fat between the head, body and tail regions of the pancreas has previously been detected (11). To the best of our knowledge, the present study is the first to investigate the association between the content and distribution of fat in the pancreas and liver of patients with T2DM. 


\section{Subjects and methods}

Subjects. A total of 70 patients with T2DM, including 54 males and 16 females, were included in the present study. Patient characteristics were as follows: Age range, 16-72 years (average, 40 years); body mass index (BMI) range, $17.36-43.09 \mathrm{~kg} / \mathrm{m}^{2}$ (average, $26.9 \mathrm{~kg} / \mathrm{m}^{2}$ ); fasting blood glucose concentrations, 7.12-22.20 mmol/l (average, $8.78 \mathrm{mmol} / \mathrm{l}$ ); glycosylated hemoglobin, 6.6-16.1\% (average, 10.61\%); and glycated albumin, 13.30-66.60\% (average, 27.65\%). Patients with T2DM were recruited in the present study if they were newly diagnosed ( $<6$ months) and had not previously undergone hypoglycemic lipid-lowering therapy. Exclusion criteria for patients with T2DM were as follows: i) The patient exhibited cardiac, liver or renal insufficiency; ii) the patient was experiencing diabetic ketoacidosis, a hyperosmolar hyperglycemic state, infection, diabetes or other acute complications; iii) pregnant and lactating women; iv) the patient was not willing to cooperate or was psychotic; and; v) the patient refused to undergo the MR examination. Cardiac insufficiency was assessed by a cardiac expert according to the ejection fraction, liver insufficiency was assessed by an expert in the Gastroenterology Department according to the level of a liver-specific enzyme, and renal insufficiency was assessed by a renal medicine specialist according to the blood levels of urea and creatinine. A total of 17 males and 14 females were included in the normal control group. Individuals were included in the normal control group if they exhibited normal blood glucose and lipid concentrations. The characteristics of the healthy volunteers were as follows: Age range, 24-59 years (average, 42.7 years); BMI, 20.81-33.58 kg/m ${ }^{2}$ (average, $25.5 \mathrm{~kg} / \mathrm{m}^{2}$ ); and fasting blood glucose concentrations, $4.21-6.13 \mathrm{mmol} / \mathrm{l}$ (average, $5.11 \mathrm{mmol} / \mathrm{l}$ ). The present study was conducted in accordance with the declaration of Helsinki, and with approval from the Ethics Committee of Beijing Friendship Hospital (Beijing, China). Written informed consent was obtained from all participants.

Inspection method. The present study used the GE Signa Excite 3.0T scanning system (GE Medical Systems, Inc., Waukesha, WI, USA) with the 8-channel phase array coil to perform cross-sectional dual-echo chemical shift imaging. The parameters were set as follows: Repetition time $=224 \mathrm{msec}$; in-phase echo time $=2.4 \mathrm{msec}$; out-of-phase echo time $=5.8 \mathrm{msec}$; flip angle $=80^{\circ}$; echo chain length $=17$; number of excitations $=2$; slice thickness $=5 \mathrm{~mm}$; and matrix size $=288 \times 192 \mathrm{~cm}$. The scanning field was set according to the size of the inspected person.

Data processing. After in-phase and out-of-phase images of the liver and pancreas were captured, they were transferred to the ADW4.2 workstation (GE Healthcare Bio-Sciences, Pittsburgh, PA, USA) for processing. The operator calculated the fat content of the liver and pancreas by determining the signal intensity of the in-phase (IP) and out of phase (OP) images at identical locations within regions of interest (ROI). The maximum intensity levels within the head, body and tail regions of the pancreas were selected as ROI. The ROI were $158.46,154.37$ and $156.47 \mathrm{~mm}^{2}$ in the head, body and tail pancreatic regions, respectively, and the average was
$155.96 \mathrm{~mm}^{2}$. The ROI were placed in the center of the measuring point in order to avoid adjacent vessels and abdominal adipose tissue. In order to measure the fat fraction (FF) of the liver, two ROI were measured at the left and right lobes of the liver. The average area of these regions was $608.17 \mathrm{~mm}^{2}$, which avoided adjacent large blood vessels and the bile duct. Each ROI was measured three times every 3-5 days and the average was calculated as the final value. The FF of the liver and pancreas was calculated using formula 1: FF=|Sip-Sopl/2Sip. In order to correct for the effects of the $\mathrm{T} 1$ and $\mathrm{T} 2$ relaxation times on the results, formula 2 was applied, according to the method outlined in a previous study (12):

$$
F F_{\text {true }}=\frac{E 1_{w}\left(E 2_{\text {ip }}(1-2 F F)-E 2_{2 \mathrm{op}}\right)}{E 2_{\text {ip }}\left(E 1_{w}-E 1_{f}\right)(1-2 F F)-E 2 \mathrm{op}\left(E 1_{w}+E 1_{f}\right)}
$$

The terms in formula 2 are defined as follows:

$$
\begin{aligned}
& E 1_{w, f}=\frac{1-e^{-T R / T 1 w, f}}{1-\cos \alpha \cdot e^{-T R / T 1 w, f}} \\
& E 2_{i \mathrm{p}, o p}=e^{-T E_{\text {in op }} / T 2^{*}}
\end{aligned}
$$

W and frepresent the water and adipose tissue content, respectively. $\alpha$ represents the excitation angle. The T1 relaxation time of the liver and pancreas in the normal tissues and organs were 809 and $725 \mathrm{msec}$, respectively. The T1 relaxation time of adipose tissue was $382 \mathrm{msec}$ in a previous study (13), and the $\mathrm{T} 2 *$ relaxation time of the pancreas and liver were 41.5 and $28.1 \mathrm{msec}$, respectively, in a previous study (14). The FF value represents the fat signal fraction calculated directly from the measured data, whereas the FFtrue value represents the fat signal fraction following correction for $\mathrm{T} 1$ and $\mathrm{T} 2 *$.

Statistical analysis. All data were analyzed using the SPSS software, version 17.0 (SPSS Inc., Chicago, IL, USA). Data are presented as the mean \pm standard deviation. The differences in the FF between the pancreatic head, body and tail regions were compared using single factor analysis of variance. The differences between the T2DM and healthy groups were compared using the Independent Samples $t$-test. The fat contents of the liver and pancreas were compared using linear correlation analysis. $\mathrm{P}<0.05$ was considered to indicate a statistically significant difference.

\section{Results}

MR chemical shift imaging quality. The MR chemical shift images clearly distinguished the contour and edges of the pancreas. The pancreatic signal intensity was uniform. With the exception of one case of absent pancreatic body and tail regions, and one case of an absent pancreatic tail, 297 ROI regions were successfully imaged. The IP/OP MR chemical shift images of the ROI were placed according to the sample (Fig. 1).

Fat content analysis. The fat contents of the pancreatic head, body and tail regions of the T2DM group were 5.59 \pm 4.70 , $4.80 \pm 3.75$ and $4.89 \pm 3.86 \%$, respectively (average, $5.19 \pm 3.75 \%$ ). The average FFtrue of the liver was $9.87 \pm 3.19 \%$. Conversely, the fat contents of the pancreatic head, body and tail regions 
Table I. Characteristics of the type 2 diabetes and normal control groups.

\begin{tabular}{lccc}
\hline Items & Type 2 diabetes group & Normal control group & P-value \\
\hline Age & $43.99 \pm 1.32$ & $42.65 \pm 9.7$ & 0.615 \\
Body mass index & $26.89 \pm 4.56$ & $25.45 \pm 2.8$ & 0.156 \\
Blood sugar & $8.78 \pm 2.99$ & $5.10 \pm 0.57$ & 0.000 \\
FFtrue & & & $3.89 \pm 2.47$ \\
Pancreas head & $5.59 \pm 4.70$ & $3.30 \pm 2.11$ \\
Pancreas body & $4.80 \pm 3.75$ & $3.23 \pm 2.23$ \\
Pancreas tail & $4.89 \pm 3.86$ & & 0.004 \\
Average FFtrue & & $3.47 \pm 2.00$ & 0.012 \\
Pancreas & $5.19 \pm 3.75$ & $7.24 \pm 2.38$ \\
Liver & $9.87 \pm 3.19$ & 0.009 \\
\hline
\end{tabular}

FFtrue, fat fraction following correction for the $\mathrm{T} 1$ and $\mathrm{T} 2{ }^{*}$ relaxation times.

$\mathbf{A}$

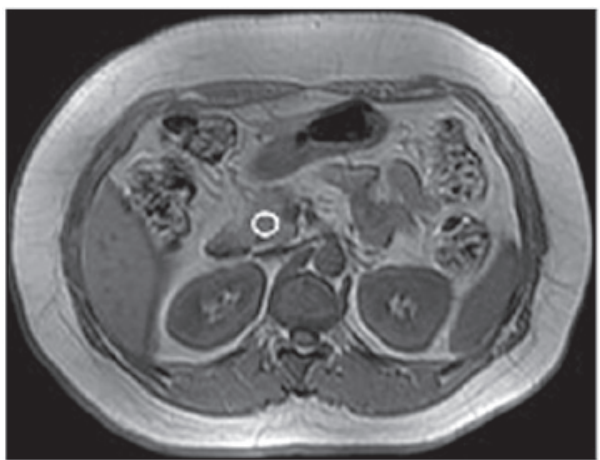

C

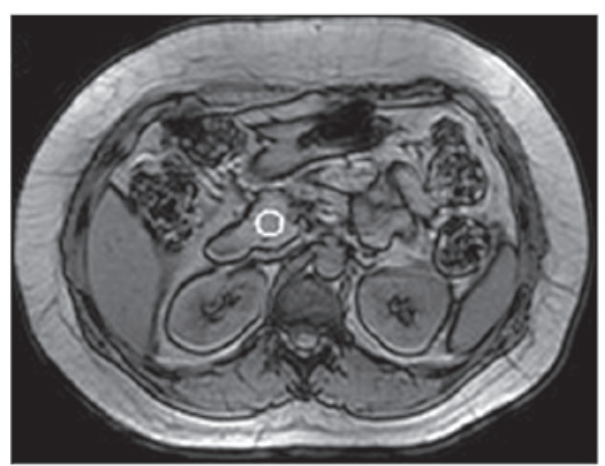

B

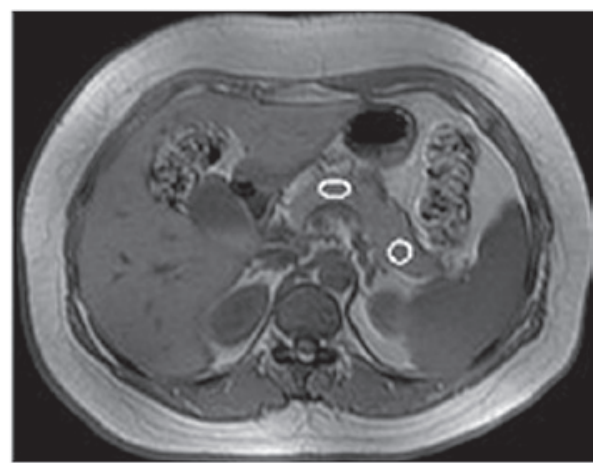

D

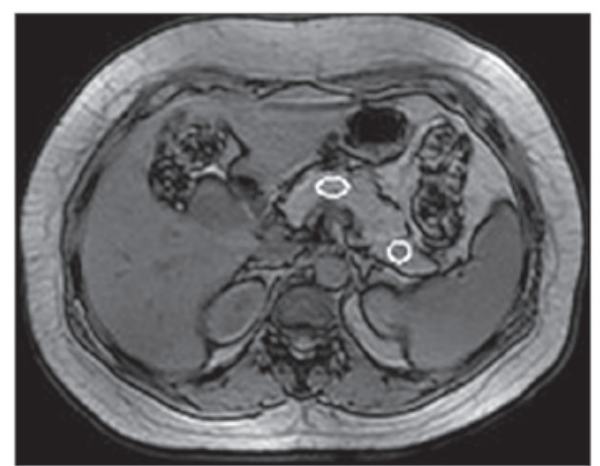

Figure 1. Measurement of the fat content of regions of interest (ROI) in the pancreas. In-phase images of ROI in the (A) pancreatic head and (B) pancreatic body and tail regions. Out of phase images of ROI in the (C) pancreatic head and (D) pancreatic body and tail regions. The same size of ROI was placed in the same position of pancreatic head. The same size of ROI was placed in the same position of interest.

in the normal control group were $3.89 \pm 2.47,3.30 \pm 2.11$ and $3.23 \pm 2.23 \%$, respectively (average, $3.47 \pm 2.00 \%$ ). Furthermore, the average FFtrue of the liver was $7.24 \pm 2.38 \%$ (Table I). There were no significant differences between the fat contents of the head, body and tail pancreatic regions in patients with T2DM ( $F=1.761 ; P>0.05)$; however, the average fat content of the pancreas in the T2DM group was significantly higher, as compared with that in the normal control group $(\mathrm{F}=3.597$; $\mathrm{P}<0.05$; Table I and Fig. 2). The correlation coefficient of the fat contents of the liver and pancreas in the T2DM group and the control group were $r=0.057$ and $r=0.337$, respectively (P>0.05; Fig. 3).

\section{Discussion}

Pancreatic fat deposition is frequently identified during an abdominal computed tomography scan or MR imaging (MRI) examination (6). Heterotopic pancreatic fat deposition has previously been associated with obesity, insulin resistance and age $(9,15)$. The distribution of pancreatic fat deposition may be uneven; however if it is confined to a region it is referred to as focal pancreatic fat deposition, and this typically occurs within the pancreatic tail and the anterior pancreatic head $(16,17)$. In a previous study, fat was shown to be widely distributed in the pancreas, and the distribution within each part of pancreas was 


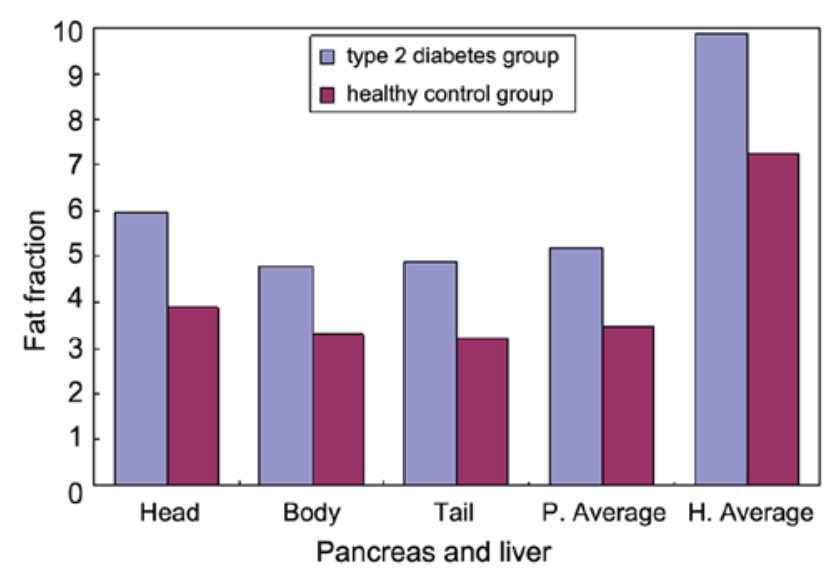

Figure 2. Average fat fraction in the pancreatic head, body and tail regions, and in the liver, of the type 2 diabetes and healthy control groups. P, pancreas; $\mathrm{H}$, hepatic.

A

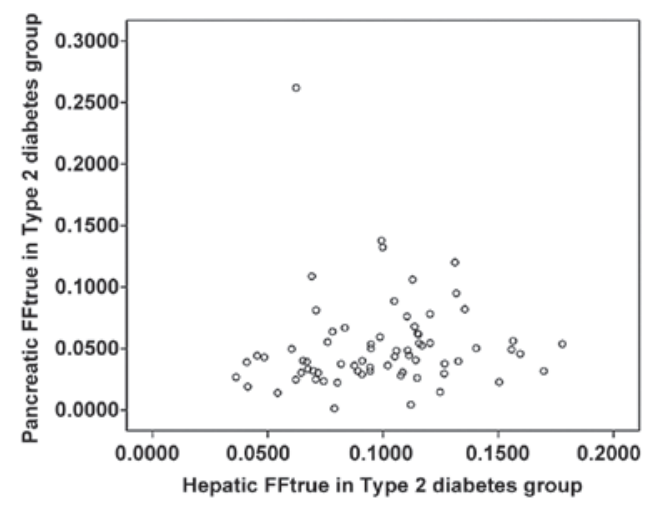

B

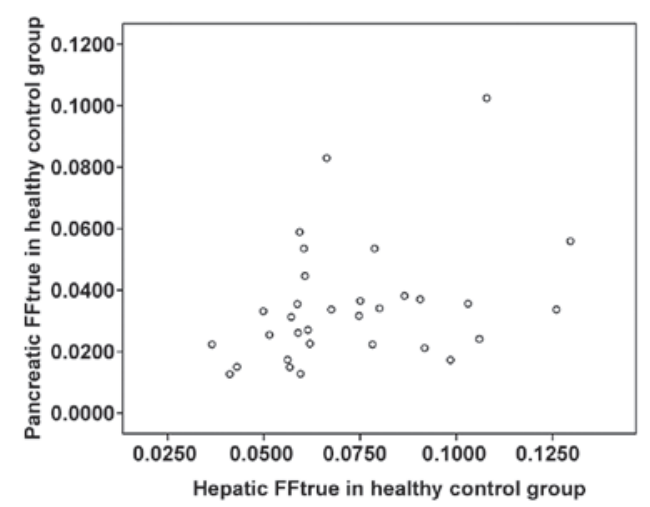

Figure 3. Correlation between the fat content of the pancreas and that of the liver in the (A) type 2 diabetes mellitus and (B) normal control groups. FFtrue, fat fraction following correction for the $\mathrm{T} 1$ and $\mathrm{T} 2 *$ relaxation times.

uniform (11). The present study demonstrated that the distribution of pancreatic fat in the head, body and tail pancreatic regions of the T2DM and normal control groups were uniform. The fat content of the pancreatic head in the T2DM group was markedly higher $(5.59 \pm 4.70 \%)$, as compared with the pancreatic body $(4.80 \pm 3.75 \%)$ and tail $(4.89 \pm 3.86 \%)$; however, there was no statistically significant difference between the three regions. These results are consistent with a previous study, in which MR chemical shift imaging was used to measure the fat content of the pancreatic head, body and tail regions in a healthy adult male (11); the authors concluded that there was no significant difference between the distribution of heterotopic fat deposition in the three regions.

In previous studies, pancreatic pimelosis was associated with obesity, type 2 diabetes and chronic pancreatitis $(8,18)$. Furthermore, the fat content of the pancreas was shown to be negatively correlated with insulin secretion in patients with impaired glucose tolerance or impaired fasting glucose (19); thus suggesting that pancreatic fat deposition may be a factor that leads to Islet $\beta$ cell dysfunction, as is consistent with the present study. In the present study, the average fat content of the pancreas in patients newly diagnosed with T2DM was $5.19 \pm 3.75 \%$, which was significantly higher, as compared with the healthy population $(3.47 \pm 2.00 \%)$. Li et al (11) reported that the average fat content of the pancreas from a healthy male with a BMI of $18-25 \mathrm{~kg} / \mathrm{m}^{2}$ was $3.03 \pm 0.59 \%$.

Tushuizen et al (20) investigated the association between the fat content of the liver and that of the pancreas in patients with T2DM and non-diabetes using MR spectroscopy, and were unable to detect a correlation between liver and pancreatic fat deposition. Similarly, Schwenzer et al (12) analyzed the association between the fat content of the liver and that of the pancreas in patients with high-risk T2DM using MRI, antiphase chemical shift imaging, and the fat selection frequency saturation method. They demonstrated that there was no significant correlation between the fat content of the liver and that of the pancreas, which is consistent with the results of the present study. However, these previous studies included various limitations, including small sample sizes and a lack of information regarding patient medical histories. Conversely, the present study selected patients that were newly diagnosed with type 2 diabetes ( $<6$ months) and had not undergone hypoglycemic lipid-lowering therapy. In addition, a larger sample size was used (70 patients in the T2DM group) and strict inclusion and exclusion criteria were applied. Therefore, the present study may state with more confidence that there was no correlation between the fat content of the liver and that of the pancreas.

The correlation between the fat content of the liver and that of the pancreas was previously investigated by van Geenen et al (21) using histological methods. Specifically, using the non-alcoholic liver disease histological grading system and the pancreatic fat infiltration grading system as references, they graded the fat content of the liver and pancreatic tissues of 80 autopsy patients. Their results demonstrated that the fat content of the pancreas was associated with that of the liver, and further suggested that the fat content of the liver was associated with that of the pancreatic lobule and overall pancreas, although it was not associated with the pancreatic interlobular fat. However, there were limitations associated with the van Geenen et al (21) study; a limited number of tissue samples were selected for histopathological examination and the fat infiltration of local tissues may not have necessarily represented the fat content of the organs. Various ROI were selected to determine the fat content by MRI examination, and the average fat content was calculated, which may have better indicated the fat content of the organs; however, MRI examination only detects the fraction of fat within a single voxel, and is unable to distinguish between fat in the pancreatic intralobule and fat in the interlobule (15).

The primary limitation of the present study was that there were numerous factors that may have influenced the 
signal detected in the dual-echo chemical shift MR images. The correction formula was applied in order to reduce the influence of the $\mathrm{T} 1$ and $\mathrm{T} 2^{*}$ relaxation times; however, heterogeneity and various other factors, including lipid sub-peaks, were unable to be eliminated and may have generated disparity in the perceived and actual fat content of the pancreas and liver, and thus requires further improvement. Future studies should focus on improving the convenience and accuracy of techniques for measuring the fat content of the pancreas and liver in patients with T2DM.

\section{Acknowledgements}

The present study was supported by the Beijing Natural Science Foundation (grant no. 7122048).

\section{References}

1. Suganami T, Tanaka M and Ogawa Y: Adipose tissue inflammation and ectopic lipid accumulation. Endocr J 59: 849-857, 2012.

2. Britton KA and Fox CS: Ectopic fat depots and cardiovascular disease. Circulation 124: e837-841, 2011.

3. Larson-Meyer DE, Newcomer BR, Ravussin E, Volaufova J, Bennett B, Chalew S, Cefalu WT and Sothern M: Intrahepatic and intramyocellular lipids are determinants of insulin resistance in prepubertal children. Diabetologia 54: 869-875, 2011.

4. Marchesini G, Bugianesi E, Forlani G, Cerrelli F, Lenzi M, Manini R, Natale S, Vanni E, Villanova N, Melchionda N and Rizzetto M: Nonalcoholic fatty liver, steatohepatitis, and the metabolic syndrome. Hepatology 37: 917-923, 2003.

5. Williams CD, Stengel J, Asike MI, Torres DM, Shaw J, Contreras M, Landt CL and Harrison SA: Prevalence of nonalcoholic fatty liver disease and nonalcoholic steatohepatitis among largely middle-aged population utilizing ultrasound and liver biopsy: A prospective study. Gastroenterology 140: 124-131, 2011.

6. Pitt HA: Hepato-pancreato-biliary fat: The good, the bad and the ugly. HPB (Oxford) 9: 92-97, 2007.

7. Wu WC and Wang CY: Association between non alcoholic fatty pancreatic disease (NAFPD) and the metabolic syndrome: Case-control retrospective study. Cardiovasc Diabetol 12: 77, 2013.

8. Sepe PS, Ohri A, Sanaka S, Berzin TM, Sekhon S, Bennett G Mehta G, Chuttani R, Kane R, Pleskow D and Sawhney MS: A prospective evaluation of fatty pancreas by using EUS 2011. Gastrointest Endosc 73: 987-993, 2011

9. Lingvay I, Esser V, Legendre JL, Price AL, Wertz KM, Adams-Huet B, Zhang S, Unger RH and Szczepaniak LS: Noninvasive quantification of pancreatic fat in humans. J Clin Endocrinol Metab 94: 4070-4076, 2009.
10. Hu HH, Kim HW, Nayak KS and Goran MI: Comparison of fat-water MRI and single-voxel MRS in the assessment of hepatic and pancreatic fat fractions in humans. Obesity (Silver Spring) 18: 841-847, 2010

11. Li J, Xie Y, Yuan F, Song B and Tang C: Noninvasive quantification of pancreatic fat in healthy male population using chemical shift magnetic resonance imaging: Effect of aging on pancreatic fat content. Pancreas 40: 295-299, 2011.

12. Schwenzer NF, Machann J, Martirosian P, Stefan N, Schraml C, Fritsche A, Claussen CD and Schick F: Quantification of pancreatic lipomatosis and liver steatosis by MRI: Comparison of in/opposed-phase and spectral-spatial excitation techniques. Invest Radiol 43: 330-337, 2008.

13. de Bazelaire CM, Duhamel GD, Rofsky NM and Alsop DC: MR imaging relaxation times of abdominal and pelvic tissues measured in vivo at 3.0 T: Preliminary results. Radiology 230: 652-659, 2004.

14. Schwenzer NF, Machann J, Haap MM, Martirosian P, Schraml C, Liebig G, Stefan N, Häring HU, Claussen CD, Fritsche A and Schick F: T2* relaxometry in liver, pancreas and spleen in a healthy cohort of one hundred twenty-nine subjects-correlation with age, gender and serum ferritin. Invest Radiol 43: 854-860, 2008.

15. Fraulob JC, Ogg-Diamantino R, Fernandes-Santos C, Aguila MB and Mandarim-de-Lacerda CA: A mouse model of metabolic syndrome: Insulin resistance, fatty liver and non-alcoholic fatty pancreas disease (NAFPD) in C57BL/6 mice fed a high fat diet. J Clin Biochem Nutr 46: 212-223, 2010.

16. Kim HJ, Byun JH, Park SH, Shin YM, Kim PN, Ha HK and Lee MG: Focal fatty replacement of the pancreas: Usefulness of chemical shift MRI. AJR Am J Roentgenol 188: 429-432, 2007.

17. Kawamoto S, Siegelman SS, Bluemke DA, Hruban RH and Fishman EK: Focal fatty infiltration in the head of the pancreas: Evaluation with multidetector computed tomography with multiplanar reformation imaging. J Comput Assist Tomogr 33: 90-95, 2009.

18. Campbell-Tofte J, Hansen HS, Mu H and Mølgaard P: Increased lipids in non-lipogenic tissues are indicators of the severity of type 2 diabetes in mice. Prostaglandins Leukot Essent Fatty Acids 76: 9-18, 2007.

19. Heni M, Machann J, Staiger H, Schwenzer NF, Peter A, Schick F, Claussen CD, Stefan N, Häring HU and Fritsche A: Pancreatic fat is negatively associated with insulin secretion in individuals with impaired fasting glucose and/or impaired glucose tolerance: A nuclear magnetic resonance study. Diabetes Metab Res Rev 26: 200-205, 2010.

20. Tushuizen ME, Bunck MC, Pouwels PJ, Bontemps S, van Waesberghe JH, Schindhelm RK, Mari A, Heine RJ and Diamant M: Pancreatic fat content and beta-cell function in men with and without type 2 diabetes. Diabetes Care 30: 2916-2921, 2007.

21. van Geenen EJ, Smits MM, Schreuder TC, van der Peet DL, Bloemena E and Mulder CJ: Nonalcoholic fatty liver disease is related to nonalcoholic fatty pancreas disease. Pancreas 39: 1185-1190, 2010. 